\title{
1 Design and Analysis of Line Transect Surveys for Primates
}

3 Stephen T. Buckland • Andrew J. Plumptre • Len Thomas • Eric A. Rexstad

$6 \quad$ S. T. Buckland (steve@mcs.st-and.ac.uk)

$7 \quad$ S. T. Buckland $\bullet$ L. Thomas $\bullet$ E. A. Rexstad

8 Centre for Research into Ecological and Environmental Modelling, University of St

9 Andrews, The Observatory, Buchanan Gardens, St Andrews, Fife KY16 9LZ, UK

10 A. J. Plumptre

11 Wildlife Conservation Society, PO Box 7487, Kampala, Uganda

13 Short title: Line transect surveys for primates

15 Corresponding author: S.T. Buckland, CREEM, The Observatory, Buchanan Gardens, St

16 Andrews, Fife KY16 9LZ, UK, +44-(0)1334-461841, steve@mcs.st-and.ac.uk 


\section{Design and Analysis of Line Transect Surveys for Primates}

20 Stephen T. Buckland • Andrew J. Plumptre • Len Thomas • Eric A. Rexstad

23 Abstract Line transect surveys are widely used for estimating abundance of primate 24 populations. The method relies on a small number of key assumptions, and if these are 25 not met, substantial bias may occur. For a variety of reasons, primate surveys often do 26 not follow what is generally considered to be best practice, either in survey design or in 27 analysis. The design often comprises too few lines (sometimes just one), subjectively 28 placed or placed along trails, so lacks both randomization and adequate replication. 29 Analysis often involves flawed or inefficient models, and often uses biased estimates of 30 the locations of primate groups relative to the line. We outline the standard method, 31 emphasizing the assumptions underlying the approach. We then consider options for

32 when it is difficult or impossible to meet key assumptions. We explore the performance

33 of these options by simulation, focusing particularly on the analysis of primate group 34 sizes, where many of the variations in survey methods have been developed. We also 35 discuss design issues, field methods, analysis, and potential alternative methodologies for 36 when standard line transect sampling cannot deliver reliable abundance estimates.

38 Keywords distance sampling • estimating primate density • line transect sampling • 39 primate surveys 


\section{Introduction}

43 Line transect sampling is a 'distance sampling' method (Buckland et al., 2001, 2004),

44 widely used for estimating the abundance of wild animal populations. The method relies

45 on a small number of key assumptions, and if these are not met, estimates of abundance

46 can have substantial bias. Line transect surveys of primates often ignore two basic

47 principles of survey design: replication and randomization. In addition, non-standard

48 methods of analysis, lacking any formal assumptions, are often employed, so that it is

49 difficult to know what can be inferred from resulting abundance estimates (Buckland et 50 al., in press). We describe line transect methods, and the assumptions on which they rely.

51 We provide guidelines for survey design and field methods to ensure better quality data,

52 and consider some analysis issues particularly relevant to primate surveys. We also

53 discuss possible alternative methods for cases where standard line transect methods are

54 expected to fail. We use a simulation study to assess different analysis approaches when

55 it is problematic to estimate group size and location, and we summarise our conclusions

56 in the discussion.

$58 \quad$ Line Transect Sampling

59 In line transect sampling (Buckland et al., 2001), lines are placed at random in the survey

60 region, or more commonly, a set of equally-spaced parallel lines is randomly

61 superimposed on the survey region. An observer walks along each line, recording any

62 animals detected within a distance $w$ of the line, together with their shortest distance from

63 the line. In some cases, the distance of detected animals from the observer (so-called

64 'radial' or 'animal-to-observer' distance), together with the angle from the line of the

65 detection, are recorded, from which the 'perpendicular' distance from the line is

66 calculated later using simple trigonometry. These perpendicular distances are used to

67 estimate a detection function, which is the probability that an animal is detected, as a 
68 function of distance from the line. For the basic method, it is assumed that this

69 probability is one at zero distance from the line; that is, animals on the line are seen with

70 certainty. Given an estimate of the detection function, we can estimate the proportion of

71 animals detected within a strip extending a distance $w$ from the line on either side. This

72 allows us to estimate animal density, by adjusting encounter rates (i.e. number of animals

73 detected per unit length of line) to allow for animals missed in this strip. Given random

74 placement of an adequate number of lines (or a grid of lines) through the survey region,

75 this density estimate is representative of the whole survey region, allowing abundance

76 within that region to be estimated.

77 Many animals, including primates, tend to occur in groups, termed 'clusters' in

78 the distance sampling literature. When these groups are well-defined, standard practice is

79 to record the group, its size, and the perpendicular distance from the centre of the group

80 to the line. Estimated density of groups is then multiplied by an estimate of mean group

81 size in the population, to obtain an estimate of animal density.

82 Survey design and analysis can be carried out using the free software Distance

83 (Thomas et al., in press).

84

85 Assumptions The key assumptions of the basic approach, with particular reference to 86 surveys of primates that occur in groups, are:

87 1. Groups whose centres are on or very close to the line are detected with certainty.

88 2. Groups are detected at their initial location, before any response to the observer. For

89 movement independent of the observer, average speed is slow relative to observer speed.

90 3. Measurement of distances from the line to the centre of each detected group is 91 accurate.

92 Two further assumptions should be emphasized as they often do not hold, or can

93 be difficult to satisfy, in primate surveys: 
94 4. There is an adequate sample of randomly-distributed lines, or a grid of lines randomly

95 positioned, in the survey region.

96 5. Group sizes are accurately recorded, at least for groups on or near the line.

97 It is important to realise that the group referred to in these assumptions is not

98 necessarily a social unit; it refers to detected animals forming a well-defined group at the

99 time of detection. This might be a group that has temporarily formed, or one part of a

100 larger social unit. In the latter case, if other parts of that unit are also detected, they are

101 recorded as separate groups.

\section{Survey Design}

104 There are two basic principles of survey design that must be met, if reliable inference on 105 population size is to be achieved. The first is randomization: if the positions of transects 106 are not random within the survey region, then there is no guarantee that they pass through 107 areas where densities are representative, and we are unable to extrapolate reliably to the

108 whole survey region. The second principle is replication. Even if the lines are random, if 109 there are too few lines, then by bad luck, they may pass through areas with atypical 110 densities. Also, precision is poorly estimated when replication is inadequate. Buckland 111 et al. (2001:232) recommend at least 10-20 lines; we would prefer closer to 20 lines than 11210 , although 10 lines of adequate length might suffice in areas where group densities vary 113 little. In practice, systematic random designs (i.e. equally-spaced lines with a random 114 start) are usually preferred to designs in which each transect is independently located at 115 random (Buckland et al., 2001:233).

116 The principles of randomization and replication both relate to assumption 4. This 117 assumption is usually not listed explicitly, because it is an aspect of survey design, which 118 is under our control - if we use an appropriate design, we guarantee that the assumption 119 is met. However, non-randomized designs (e.g. transects along trails) with inadequate 
120 replication (fewer than 10 lines) are frequent in primate surveys, so we state the

121 assumption explicitly here. If transect lines are not positioned randomly, but instead are

122 located on trails, then the burden of proof falls on the researcher to demonstrate that the

123 selected trails provide a representative sample of the population, and that the distribution

124 of animals within the surveyed strip is uniform with respect to distance from the line.

125 We show four different strategies for designing a survey (Fig. 1): a

126 straightforward systematic design with a random start (Fig. 1(a)); a design with two

127 strata, with a systematic random sample of lines in each stratum, and higher sampling

128 intensity in one of the strata (Fig. 1(b)); short line segments, spaced so that the separation

129 distance between successive segments on the same line is the same as the distance

130 separating successive lines, which ensures a systematic grid of line segments through the

131 region (Fig. 1(c)); a design based on a systematic grid of points through the survey

132 region, with a circuit (square) of transect lines located around each point (Fig. 1(d)). This

133 last design has the advantage that the observer can start from any location on the circuit

134 (e.g. where access is easiest, such as an intersection of the circuit with a track or trail),

135 and finishes at the same place. However, there is a risk of disturbance of animals on one

136 section of the circuit when the observer is covering another section. If this is thought to

137 be an issue, gaps can be introduced at each corner of the circuit, to separate out the 138 sections.

139 All four designs have lines that are evenly spread through the survey region (Fig.

140 1). Survey effort is not clustered in areas of easier access, for example. If there are parts

141 of the survey region that are costly to survey, the region can be divided into strata, with a

142 randomized design in each stratum, but with a lower sampling effort in strata that are

143 more costly to survey (Fig. 1(b)). Such a design allows unbiased estimation of primate

144 abundance, whereas subjective placement of lines related to ease of access may generate

145 substantial bias. Usually, we assume that systematically-spaced lines are in fact 
146 independently randomly located in our analyses. Typically, systematic samples yield

147 better precision than simple random samples, especially if there are strong trends in

148 density through the region, but it is more difficult to estimate that precision. Fewster et

149 al. (2009) show that a post-stratification strategy can yield good estimates of the

150 systematic sampling variance.

151 Survey design is discussed in depth by Buckland et al. (2001:228-323) and by

152 Strindberg et al. (2004). Karanth and Nichols (2002:87-120) discuss survey design and

153 field methods for line transect surveys of tropical forest-dwelling ungulates, which share

154 many of the issue associated with primate surveys. A useful training video is also freely

155 available online (http://www.youtube.com/monitoringtigers).

156

157 Field Methods

158 As noted above, a key assumption is that lines are placed at random, independently of 159 animal locations. This often necessitates cutting of vegetation, which should be minimal

160 when it is required, and ideally carried out at least one week before the line is surveyed,

161 by which time there should be no lasting effect from disturbance. If cutting is sufficient

162 only to allow quiet passage and facilitate data collection, then disturbance of the animals

163 while surveying will be minimized without creating marked highways. Obvious cut

164 transects may affect animal behaviour and distribution, and give easy access to hunters,

165 for example, who would influence detection probability and encounter rates, making

166 them unrepresentative of the larger survey area. Note that it is not essential that

167 observers walk exactly on the transect line - they can leave it, for example to move

168 around small obstacles if this minimizes cutting, so long as detection of animals on the

169 line is still certain. However, the measured distances must be of detected animals from

170 the line, and not from the route taken by the observer, if this differs. Although it is often 
171 much less costly to conduct surveys along trails, there can be no guarantee that densities

172 (or temporal trends in density) along trails are representative.

173 Repeat surveys of the same line within a season to increase sample size is sensible

174 and often essential, but these must not then be analysed as if different lines had been

175 surveyed; the transect should be entered in the software Distance, with effort recorded as

176 line length times the number of times the line was surveyed.

177 There should be a clear protocol so that fieldworkers can determine what

178 constitutes a group for the purposes of the survey. For example, if animals are separated

179 by more than say $20 \mathrm{~m}$ from the originally detected group, the protocol might state that

180 these should be treated as a second group. This might result in one large social unit being

181 recorded as many groups. Any of those groups that is detected and whose centre is

182 located within the survey strip of half-width $w$ should be recorded, and their distance

183 from the line measured or estimated.

184 Distances of group centres from the line should be measured as accurately as

185 possible (assumption 3). This requires that the position of the line is well-defined, so that

186 distances from the line are well-defined. Unless distances are sufficiently small to be

187 measured with a tape without undue disturbance or delay, a laser rangefinder should

188 always be used for primate surveys. It may not always be possible to take a direct

189 measurement, for example because of intervening vegetation, but it is possible to take

190 several measurements to visible objects (e.g. tree trunks) by moving off the transect and

191 summing the distances that form the perpendicular distance you need to measure. The

192 ability to check distances to visible objects by rangefinder is invaluable for improving

193 estimates of distances. We tested five field assistants who regularly census primates and

194 measure perpendicular distances in Uganda in 2008 for their ability to estimate distance

195 by eye (29 obs) and with a laser rangefinder (80 obs), and compared these with the

196 measured distance using a tape (true value). With a rangefinder, 62\% of observations 
197 were exact (when measured to the nearest metre), 91\% were within $1 \mathrm{~m}$, and 97\% within

$1982 \mathrm{~m}$ of the true distance up to distances of $40 \mathrm{~m}$. Only $7 \%$ of the estimates by eye were

199 exact, with 24\% within $1 \mathrm{~m}$ and 59\% within $2 \mathrm{~m}$. Some estimates by eye were up to 13

200 m away from the true value. There was also a bias towards underestimating true distance

201 by eye with $68 \%$ less than or equal to the true value and $38 \%$ greater than or equal to the

202 true value (A. J. Plumptre, unpublished data). This bias would artificially increase

203 estimates of primate density. Field aids such as rangefinders are inexpensive, especially

204 when compared with the costs resulting from poor abundance estimates.

205 Primates are often in large, dispersed groups, so that it is difficult to estimate

206 distance except for the animals first detected. The problem is made worse if the animals

207 flee from the observer. Given the difficulty in estimating the location of a group centre, it

208 is common practice to record the distance from the line of the first animal detected from a

209 group, and to assume that distance is the distance to the group centre (Struhsaker, 1981;

210 Hassel-Finnegan et al., 2008). Of course, the first animal detected tends to be closer to

211 the observer, and hence closer to the line, than the centre of the group (Marshall et al.,

212 2008). The measured distances are therefore systematically biased downwards, which

213 artificially inflates density estimates. This source of bias is well-known (e.g. Whitesides

214 et al., 1988; Marshall et al., 2008), yet the practice persists, and as a consequence,

215 standard line transect sampling is considered to overestimate density in the primate

216 literature (Hassel-Finnegan et al., 2008).

217 Where it is impossible to determine location of group centres with sufficient 218 accuracy, but feasible to estimate distances to each detected animal, then a solution exists

219 (Buckland et al., 2001:75-76). The methods in Distance are extremely robust to the 220 assumption that detections are independent events, which is why we do not list this as a

221 key assumption. As a consequence, you can ignore the existence of groups when using

222 line transect sampling to estimate density or abundance. Each individual animal that is 
223 detected is recorded, along with its distance from the line. This may compromise ability

224 to measure distances accurately, but approximate estimates of distance, coupled with

225 observer training, is preferable to accurate measurements of the wrong distance. The

226 approach would usually be impractical if a tape is used to measure distances, but is more

227 feasible if a laser rangefinder is used. The task can be made more practical by defining a

228 maximum distance from the line beyond which detected animals are not recorded; this

229 distance would then be used as the truncation distance $w$ for analysis.

230 Adopting this approach, it does not matter if observers fail to detect some animals

231 in a detected group, and assumption 5 above can be dropped. The method assumes,

232 however, that all animals on or very close to the line are detected. If an animal is

233 detected but cannot be accurately located (e.g. because it is well away from the line, and

234 is heard but not seen), it can be excluded from the sample; this just changes the meaning

235 of the detection function slightly, in that it now estimates the probability that an animal is

236 both detected and accurately located, as a function of distance from the line. This does

237 not generate bias in density estimates, provided all those on the line are detected and 238 recorded.

239 If it is not feasible to record all detected individuals, together with their distances

240 from the line, then it is important to estimate the size and location of detected groups as

241 accurately as possible. In fact, bias in estimates of the size or location of groups well

242 away from the line need not be problematic (see next section), but for those groups on or

243 close to the line, bias should be as small as possible. A field protocol should be

244 developed with these issues in mind. For example if animals do not respond to observers,

245 observing the group for a period of time from different locations on and off the line may

246 allow an accurate assessment of size. If animals do respond, a quick count may be

247 needed, and multiple observers with slightly different vantage points, and a well- 
248 rehearsed protocol for coordinating their count (e.g. sketches of animal locations together

249 with arrows to indicate direction of movement), may be effective.

250 If neither of these strategies is achievable, it may be necessary to estimate mean 251 group size and spread in a separate study from the line transect survey. In this case, the

252 study should be conducted synchronously with the line transect survey. In this way, the

253 mean size and spread of groups in the study should be comparable with the mean size and

254 spread in the population at the time of the line transect survey; variation in size and

255 spread by time of day, season or other factors (Plumptre, 2000) will be controlled for.

256 Problems with this approach are a) it may be difficult to achieve an adequate sample size

257 - at least 10, and preferably nearer 20, especially if group size is very variable; b) if only

258 habituated groups can be monitored in this way, they may not be representative of all 259 groups; and c) it is still necessary to estimate the location relative to the line of groups

260 detected during the line transect survey. To address this last point, it may be necessary to 261 record the distance to the closest animal (whether it is closest to the line or to the

262 observer), and correct either the recorded distances or the effective strip half-width

263 (Whitesides et al., 1988). Hassell-Finnegan et al. (2008) argued against this strategy

264 because group shape is usually not circular making spread difficult to quantify. To allow

265 for this, you could estimate group spread as the average of several values, recorded using 266 diameters across the group at different orientations. Whitesides et al. (1988) defined

267 group spread as the radius of the circle that has the same area as the area occupied by the 268 group; given a means to estimate this area, we can thus estimate group spread.

\section{Data Analysis}

271 Standard line transect analyses are usually conducted using the software Distance

272 (Thomas et al., in press). There are three components to estimation when animals occur

273 in groups. The first is encounter rate, which is the number of groups detected per unit 
274 length of transect (excluding those whose centres are further from the line than the 275 truncation distance $w$ ). The second is the estimated proportion detected of those groups 276 whose centres are within distance $w$ of the line. The third is the estimate of mean group 277 size in the population. Typically, this is smaller than the mean size of detected groups, 278 because larger groups are more detectable. However, group sizes may be 279 underestimated, as it is difficult to detect all animals within a group, so the mean of 280 recorded group sizes might be biased high or low if used as an estimate of mean group 281 size in the population. The default method of estimating mean group size in software 282 Distance, in which the logarithm of group size is regressed on estimated probability of 283 detection as a function of distance from the line, corrects for both sources of bias, 284 although if there is bias in the recorded size of groups on or near the line, the correction 285 will be partial. Buckland et al. (2001) give a detailed account of analysis methods.

286 A possible departure from the standard analysis is to record distance from the line 287 of the nearest animal only, and then to correct for bias at the analysis stage. Whitesides 288 et al. (1988) added half the mean group spread, $\bar{r}$, to the estimated effective half-width 289 of the strip, $\hat{\mu}$. (The effective strip half-width $\mu$ is the distance from the line at which as 290 many groups are detected beyond $\mu$ as are missed within $\mu$ of the line (Buckland et al., 291 2001:3).) This method is unsatisfactory when a group straddles the line. For example if 292 the nearest animal was recorded as on the line, then adding half the mean group spread 293 gives a distance of $\bar{r}$, but a group at this distance from the line is not expected to straddle 294 the line. For the data of Whitesides et al. (1988), the mean group spreads were larger 295 than the effective strip half-width for five of the seven species. Thus most groups whose 296 centres were within the effective strip half-width of the line would be expected to straddle 297 the line. A better approach would appear to be to correct individual distances. Suppose, 298 for example, that for a given group, the distance from the line of the nearest animal to the 
299 line is recorded, along with whether the group straddled the transect. At the analysis

300 stage, for those groups that do not straddle the transect, half the mean group spread

301 should be added to the recorded distance. For those groups that do straddle the line, we

302 could assign a distance from the line by selecting a value at random from a uniform

303 distribution between zero and half the group spread. If it is assumed that the recorded

304 distance is of the nearest animal to the observer, then the correction to individual

305 distances that Whitesides et al. (1988) developed for fitting the hazard-rate model can be

306 adopted: the corrected perpendicular distance is equal to the recorded perpendicular

307 distance multiplied by $1+\bar{r} / A O D$ where $\bar{r}$ is half the mean group spread and AOD is the

308 animal-to-observer distance. This is based on the premise that the distance from the

309 observer to the group centre should on average be the distance from the observer to the

310 nearest animal plus the mean group spread, and simple trigonometry shows that the

311 multiplicative correction for the perpendicular distance is the same as that for the animal-

312 to-observer distance. If there are many recorded perpendicular distances of zero, it may

313 be preferable to record whether a group straddles the line; for those that do not, apply the

314 above correction, while for those that do, take the perpendicular distance to be a random

315 value from the uniform distribution on $(0, \bar{r})$.

\section{Alternative Methods}

318 In some circumstances, it may prove impossible to meet the assumptions of standard line

319 transect sampling to an adequate approximation. Other approaches should then be 320 considered.

321 If it is feasible to record each individual animal that is detected, together with its

322 distance from the line, but it is thought that some animals on the line are missed, it may

323 be possible to conduct trials by locating animals, perhaps using radio collars, and then 
324 sending observers who are ignorant of animals' positions past the animals at a known

325 closest distance of approach. These trials result in binary data, where one corresponds to

326 detection by the observer, and zero corresponds to non-detection. These data may be

327 modelled using logistic regression, with distance from the line and possibly other

328 variables as covariates, from which the probability of detection on the line (i.e.

329 distance=0) may be estimated. If there are any covariates other than distance in the

330 model, this estimate will be a mean value across the trial groups, for which the

331 probability will vary according to the values of the covariates. This estimate and its

332 standard error may then be included as a multiplier in the Distance software, when

333 analysing the line transect survey data. Similarly, if groups rather than individuals are

334 recorded, but some groups on the line may be missed, trials might be set up involving the 335 group rather than an individual animal.

336 Another distance sampling approach that may work for primates that call is cue 337 counting, as implemented for birds by Buckland (2006). The design comprises a grid of

338 points. An observer stands at each point for a predetermined time, and records any calls

339 heard during this time, together with an estimate of the distance of the calling animal

340 from the point. Cue rate (number of calls per animal per unit time) is estimated in a

341 synchronous survey, to allow conversion from number of calls per unit area per unit time

342 to estimated animal density. Movement of animals independent of the observer does not

343 bias this method, and silent animals above the point need not be detected. Instead, we

344 assume that a call is certain to be heard if the animal is above the point. The

345 disadvantages of this approach are that it can be difficult to estimate distances to calling

346 animals, and it is difficult to ensure that a representative sample of animals is monitored

347 to estimate the cue rate.

348 If animals can be lured in by playing a call, then lure strip transects may be 349 possible, as implemented in a recent study of cotton-top tamarins Saguinus oedipus 
350 (Savage et al., in prep.). Observers simultaneously travel along two parallel transects,

351 luring animals from within the strip between the transects. If the lure causes animals to

352 respond by calling, but does not attract them in, a line transect version of this approach

353 might be workable, with just one transect at each location. If several observers are

354 positioned along the line, distances of responding groups from the line may be estimated

355 by triangulation (B. Rawson, pers. comm.). Another possibility is lure point transects

356 (Buckland et al., 2006), in which trials are conducted on animals with known location,

357 and from which a model for the detection function is fitted using logistic regression; this

358 function represents the probability that an animal will be detected from the point at which

359 the lure is played. This detection function model is then assumed to hold for the main

360 survey, where a lure is played at each of a number of points systematically spaced

361 through the survey region.

362

\section{Simulation study}

364 Buckland et al. (in press) conducted a simulation study to assess the performance of

365 methods based on animal-to-observer distances. We use the same simulation set-up to

366 assess several analysis options for survey data on primate groups based on standard line

367 transect methods. Details of how the data in simulation set A were generated are given

368 by Buckland et al. (in press). The set comprises 100 datasets for each combination of

369 three mean group sizes (3, 10 or 30), three half-group spreads (10 m, $25 \mathrm{~m}$ or $50 \mathrm{~m})$, three

370 densities (15, 50 or 150 groups $\mathrm{km}^{-2}$ ) and two detection functions, making 1800

371 simulated populations in all, each of which was surveyed once. The two detection

372 functions are given by two parameterizations of the hazard-rate model:

$373 g(y)=1-\exp \left\{-(y / 20)^{-2}\right\}$ and $g(y)=1-\exp \left\{-(y / 30)^{-4}\right\}$. The hazard-rate model was

374 used because it has an underlying model for the detection process (Hayes and Buckland, 
375 1983), whereas other models are simply proposed shapes. This allows animal-to-

376 observer distances to be generated along with perpendicular distances. In the first,

377 detection is certain out to $10 \mathrm{~m}$, and declines to 0.2 at just over $40 \mathrm{~m}$; in the second,

378 detection remains certain to greater distances (around 25m) but then drops more rapidly,

379 again falling to 0.2 at just over $40 \mathrm{~m}$ (Buckland et al., in press, Fig. 1). If at least one

380 animal in a group was detected, remaining undetected animals were given an enhanced

381 probability of detection, by simulating a second 'pass' with the scale parameter of the

382 detection function increased by 50\% (Buckland et al., in press). The number of groups

383 detected was typically in the range 60-120 for each population.

384

385 Estimating Densities

386 We used the software Distance to estimate density and mean group size in the population.

387 We set truncation distances $w$ (Buckland et al., 2001:103-108) so that around 10\% of

388 observations were truncated. We considered only two possible detection function

389 models: the half-normal key with cosine adjustments, and the uniform key with cosine

390 adjustments (Buckland et al., 2001). We used Akaike’s Information Criterion (AIC) to

391 select any adjustment terms, and to select between the two keys. We did not use the true

392 detection function (the hazard-rate model) in analysis, as we wished to assess

393 performance of the method using an approximating model; when analysing real data, we

394 would not know the true model. For each dataset, we implemented the following 395 methods for extracting distances for analysis.

397 1. Perpendicular distances from the line to each individual animal detected, as if the 398 animals did not occur in groups. Truncation distance $w$ was $50 \mathrm{~m}$ for all analyses.

399 2. Perpendicular distances from the line to group centres, where we determined group 400 size and group centre only from detected animals in the group, defining group centre as 
401 the mean of perpendicular distances of detected animals from the line. Truncation

402 distance $w$ for group centres was $75 \mathrm{~m}$ for mean group size of 3, $100 \mathrm{~m}$ for mean group

403 size of 10 , and $125 \mathrm{~m}$ for mean group size of 30 .

404 3. Perpendicular distances from the line to group centres, where we assume that true

405 group size and location are known. Truncation distance $w$ as for method 2.

406 4. Perpendicular distances from the line to groups, where we take group location as the

407 location of the first animal detected from the group, and we estimate group size as in

408 method 2. Truncation distance $w$ as for method 2.

409 5. Perpendicular distances from the line to groups, where we take group location as the

410 location of the first animal detected from the group, but true group size is known.

411 Truncation distance $w$ as for method 2.

412

413 We implemented method 3 to act as a gold standard, to compare with methods

414 that can be achieved in practice. Similarly, we implemented method 5 to allow us to

415 separate the effect of recording group location as the location of the first animal detected

416 from the effect arising from underestimating group size.

417 This is intentionally an idealized study, with a large sample of lines systematically

418 spaced with a random start, and most key assumptions satisfied. If methods perform

419 poorly here, they can certainly be expected to in real studies. We conducted further

420 simulations (simulation set B) that were far more challenging with respect to groups.

421 First, we made group size much more variable. We achieved this by setting $p=0.5$

422 instead of 0.75 in the model of Buckland et al. (in press) for controlling variability in

423 group size; the further below one that we set $p$, the greater the variability. If the mean

424 group size is 10 , this choice of $p$ generates about one group in 200 with a size greater 425 than 100. Second, we made detection of individuals in a group independent, omitting the 
426 second pass described by Buckland et al. (in press). The effect of this is that recorded

427 group sizes tend to be much smaller than true group sizes, especially for groups located

428 further from the line, or with large group spread. This adversely affects methods that use

429 recorded group size rather than true size (methods 1, 2 and 4).

430

431 Results

432 The hazard-rate model has a very flat shoulder for the values of the shape parameter used

433 in this study, which means that the detection probability, assumed to be one at zero

434 distance, remains at one for some distance from the line, before it starts to fall (Fig. 1 of

435 Buckland et al., in press). Neither of the detection function models used for analysis

436 (either a half-normal or a uniform key, with cosine adjustments) share this property. As a

437 consequence, we anticipated modest upward bias in density estimates from this source.

438 Method 3 is based on having perfect knowledge of detected groups, and a priori, we

439 expected this method to perform best. It gave consistent estimates of density with good

440 precision, and some positive bias $(+8.6 \%)$, as anticipated (Table 1$)$. We see also that

441 method 1 (average bias $+8.0 \%$ ), based on analysing individuals, matches the performance

442 of method 3. This is surprising, as method 3 uses additional information not available to

443 method 1: the true number of animals in a detected group, and the mean location of all

444 animals in a detected group. Plumptre and Cox (2006) proposed the use of method 2, but

445 its performance was disappointing, with bias tending to increase with increasing group

446 size and group spread. Bias also differed between the two detection functions. Method 4

447 showed inconsistent biases. Biases were smaller when the true detection function was

448 given by $g(y)=1-\exp \left\{-(y / 20)^{-2}\right\}$ than when it was given by

$449 g(y)=1-\exp \left\{-(y / 30)^{-4}\right\}$. For large groups, bias was a decreasing function of group 450 spread for the second of these detection functions, but an increasing function for the first. 
451 Method 5 allows us to assess the effect of using distance to the first animal detected in

452 the absence of bias in group size estimation. We see the anticipated positive bias, which

453 increases as group spread increases. All five methods show substantially lower bias on

454 average than the 'modified Kelker' methods based on animal-to-observer distances

455 assessed by Buckland et al. (in press).

456 The default group size regression method of Distance, in which log group size is 457 regressed on the estimated detection probability, reduces but does not entirely remove the 458 bias arising from estimating true group sizes by the recorded group sizes (Table 2). The 459 mean of true sizes of detected groups tends to overestimate the mean of groups in the 460 population, as a result of size bias: groups with many animals are more likely to be 461 detected than groups with few animals. The size bias is relatively modest here (ranging 462 between around $+1 \%$ and $+10 \%$ ). The regression method reduces the bias at the cost of

463 increased variance. However, the contribution of this variance to the overall variance in 464 the density estimate is small, so that the increased variance has minimal impact.

465 We show results for the scenarios in which group size was highly variable, and 466 for which recorded group sizes were much smaller on average than true sizes (simulation 467 set B, Table 3). We find that methods 1 and 3 maintain their good performance, while 468 that of the other methods deteriorates.

\section{Discussion}

471 If, having detected a group of primates, it is possible to detect all animals in the group, 472 and to estimate the distance of the group from the line, then method 3 can be expected to 473 provide good estimates of density with low bias. The centre of the group can be defined 474 in a way that makes it easier to estimate, provided there is not systematic bias of the type 475 that occurs if distance to the first detected animal is used. Thus if it is not possible to 476 estimate the mean distance from the line of animals in the group, it may be possible to 
477 estimate the distance from the line of the left-most animal and of the right-most animal,

478 and at the analysis stage, to calculate the mid-point between them as the distance of the

479 group from the line. If the left-most animal is to the left of the line and the right-most

480 animal to the right (i.e. the group straddles the line), then care must be taken to record

481 one of the distances as negative, before taking the average.

482 If group size and location cannot be determined with good accuracy, the strategy

483 of recording each detected animal as if it were a separate detection, together with the

484 distance of each detected animal from the line, gives equally good estimates of density.

485 Because detections are not independent in this case, AIC tends to select too many terms

486 for the detection function, and goodness-of-fit tests tend to generate spurious significant

487 results, indicating poor fit when in fact the model is adequate (Buckland et al, 2001:76).

488 In the simulation study, we selected the model chosen by AIC, yet despite obvious

489 overfitting in some cases, estimation was still good.

490 Upward bias in line transect sampling can occur from a source other than the

491 recording of biased measurements of distance. For relatively mobile species, their

492 average speed of non-responsive movement may be similar to that of the observer,

493 especially given that observers often deliberately walk very slowly and quietly, to avoid

494 disturbance, and to increase detection probabilities. If the average speed of movement is

495 under half that of the observer, bias is negligible, but bias increases as average speed of

496 the groups increases (Buckland et al., 2001:31).

497 Responsive movement of animals can compromise data quality. If a large group

498 of animals flushes simultaneously in response to the observer, it can be impossible to

499 record distances to each individual animal that is detected. Failure to record all distances

500 from the line of detected animals away from the line is not a problem, as it is perfectly

501 acceptable to model the combined probability of detecting and accurately positioning the

502 animal. If some groups respond by flushing while others do not, the detection function 
503 will vary by group, but pooling robustness (Buckland et al., 2004:389-392) means that

504 this does not bias the method. More problematic are groups on or close to the line. If it 505 is possible to estimate approximate distances from the line to the location of each animal 506 prior to flushing, this should be done. If not, then it may be necessary to record the 507 group, rather than each animal in the group. In this case, it will be necessary to estimate 508 the distance of the group centre from the line, and to estimate the size of the group. If 509 neither of these options is feasible, but the group is formed of smaller 'sub-groups' of 510 animals, then it may be possible to record each detected sub-group, together with its size 511 and the distance of its centre from the line. In this case, it is not necessary to detect every 512 sub-group in a group, provided all those on or very close to the line are detected.

513 If it truly is impractical to estimate the density of primates using distance 514 sampling with direct observations, then consideration could be given to employing so515 called 'indirect estimation' techniques. With these methods, distance sampling is used to 516 estimate the density of sign, such as nests or dung, left by the primate population.

517 Additional parameters related to the rate of appearance and disappearance of sign need to 518 be estimated to permit conversion of sign density to animal density. For an excellent 519 review of survey methods for great apes, for which nest surveys are common, see Kühl et 520 al. (2008).

521 Estimation of density for many primate populations represents a great challenge.

522 However, applications to other taxa are often no less challenging. For example, surveys 523 of whales have to address the problems of very low densities of animals across large 524 regions, in an environment where distances are difficult to estimate, with the possibility 525 of responsive movement before detection, and often with no certainty that an animal on 526 the line will be detected. After over 30 years of active development, distance sampling 527 methods can now be reliably applied to many species. We hope that this paper will help 528 researchers to achieve more reliable estimation of the size of primate populations. 


\section{ACKNOWLEDGEMENTS}

531 We thank Anne Savage, Ben Rawson, two referees and the editor for their constructive

532 comments, which led to a much-improved paper.

533

\section{REFERENCES}

535 Buckland, S. T. (2006). Point transect surveys for songbirds: robust methodologies. The 536 Auk, 123, 345-357.

537 Buckland, S. T., Anderson, D. R., Burnham, K. P., Laake, J. L., Borchers, D. L., \&

538 Thomas, L. (2001). Introduction to Distance Sampling. Oxford: Oxford University 539 Press.

540 Buckland, S. T., Anderson, D. R., Burnham, K. P., Laake, J. L, Borchers, D. L., \&

541 Thomas, L. (Eds). (2004). Advanced Distance Sampling. Oxford: Oxford University 542 Press.

543 Buckland, S. T., Plumptre, A. J., Thomas, L., \& Rexstad, E. A. (in press). Line transect 544 sampling of primates: can animal-to-observer distance methods work? Int J Primatol.

545 Buckland, S. T., Summers, R. W., Borchers, D. L., \& Thomas, L. (2006). Point transect 546 sampling with traps or lures. J App Ecol, 43, 377-384.

547 Fewster, R. M., Buckland, S. T., Burnham, K. P., Borchers, D. L., Jupp, P. E., Laake, J. 548 L., \& Thomas, L. (2009). Estimating the encounter rate variance in distance sampling. 549 Biometrics, 65, 225-236.

550 Hassel-Finnegan, H. M., Borries, C., Larney, E., Umponjan, M., \& Koenig, A. (2008).

551 How reliable are density estimates for diurnal primates? Int J Primatol, 29, 1175-1187.

552 Hayes, R. J., \& Buckland, S. T. (1983). Radial distance models for the line transect 553 method. Biometrics, 39, 29-42. 
554 Karanth, K. U. \& Nichols, J. D. (Eds). (2001). Monitoring Tigers and their Prey.

555 Bangalore, India: Centre for Wildlife Studies.

556 Kühl, H., Maisels, F., Ancrenaz, M., \& Williamson, E. A. (2008). Best Practice

557 Guidelines for Surveys and Monitoring of Great Ape Populations. Gland, Switzerland:

558 IUCN SSC Primate Specialist Group.

559 Marshall, A. R., Lovett, J. C., \& White, P. C. L. (2008). Selection of line-transect

560 methods for estimating the density of group-living animals: lessons from the primates.

561 Am J Primatol, 70, 1-11.

562 Plumptre, A. J. (2000). Monitoring mammal populations with line transect techniques in

563 African forests. J App Ecol, 37, 356-368.

564 Plumptre, A. J., \& Cox, D. (2006). Counting primates for conservation: primate surveys

565 in Uganda. Primates, 47, 65-73.

566 Savage, A., Thomas, L., Leighty, K., Soto, L., Causado, J., \& Medina, F. (in prep.).

567 Innovative census techniques estimate the cotton-top tamarin (Saguinus oedipus)

568 population in Colombia.

569 Strindberg, S., Buckland, S. T., \& Thomas, L. (2004). Design of distance sampling

570 surveys and Geographic Information Systems. In S. T. Buckland, D. R. Anderson, K. P.

571 Burnham, J. L. Laake, D. L. Borchers \& L. Thomas (Eds), Advanced Distance Sampling

572 (pp. 190-228). Oxford: Oxford University Press.

573 Struhsaker, T. T. (1981). Census methods for estimating densities. In National Research

574 Council, Techniques for the study of primate population ecology (pp. 36-80).

575 Washington: National Academy Press.

576 Thomas, L., Buckland, S. T., Rexstad, E. A., Laake, J. L., Strindberg, S., Hedley, S. L.,

577 Bishop, J. R. B., Marques, T. A. \& Burnham, K. P. (in press). Distance software: design

578 and analysis of distance sampling surveys for estimating population size. J App Ecol. 
579 Whitesides, G. H., Oates, J. F., Green, S. M., \& Kluberdanz, R. P. (1988). Estimating 580 primate densities from transects in a west African rain forest: a comparison of techniques.

$581 J$ Anim Ecol, 57,345-367.

582

583 Figure legend

584

585 Fig. 1. Examples of survey design within a region for which an estimate of abundance is 586 required. (a) Systematic random sample of lines that span the survey region. (b) 587 Stratified systematic sample of lines that span the stratum. (c) Systematic sample of line 588 segments. The design comprises the solid line segments. (d) Systematic sample of 589 circuit transects.

590

591

592 
593 Table 1. Percent bias of density estimates for the five methods of estimation for 594 simulation set A. Coefficients of variation of estimates, expressed as percentages, are 595 shown in parentheses. Method 1: analysis of individual detections in Distance. Method 596 2: analysis of groups in Distance, group size and centre location based on detected 597 animals only. Method 3: analysis of groups in Distance, true group size and centre 598 location known. Method 4: analysis of groups in Distance, group size based on detected 599 animals only, group location taken as location of first detected animal. Method 5: 600 analysis of groups in Distance, true group size known, group location taken as location of 601 first detected animal.

602

603

\begin{tabular}{lccccccccc}
\hline Mean group size & & 3 & & & 10 & & & 30 \\
Half-group spread & $10 \mathrm{~m}$ & $25 \mathrm{~m}$ & $50 \mathrm{~m}$ & $10 \mathrm{~m}$ & $25 \mathrm{~m}$ & $50 \mathrm{~m}$ & $10 \mathrm{~m}$ & $25 \mathrm{~m}$ & $50 \mathrm{~m}$ \\
True density & 15 & 15 & 15 & 50 & 50 & 50 & 150 & 150 & 150 \\
\hline
\end{tabular}

607

608

609

610

611

612 Method 3

613

614

615

616

617

618

619

Method 1

Method 2

Method 4

Method 5

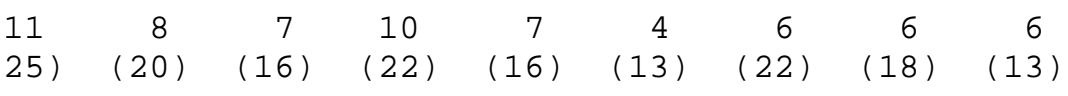

$\begin{array}{lllllllll}17 & 16 & 24 & 15 & 19 & 31 & -10 & 7 & 48\end{array}$

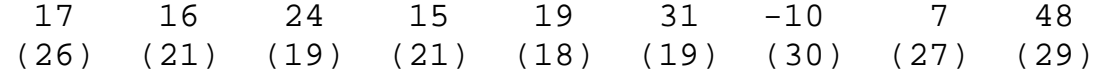

620

621

622

623

624

625

626

627

628

629

630

631

$\begin{array}{lllllllll}9 & 5 & 1 & 6 & 8 & 5 & 2 & 7 & 6\end{array}$

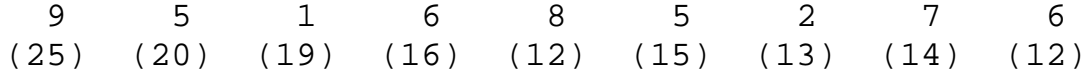

$\begin{array}{lllllllll}15 & 11 & 9 & 14 & 14 & 11 & -10 & -1 & 11\end{array}$

Method 5

$\begin{array}{llllllll}(25) & (20) & (20) & (20) & (17) & (18) & \text { (29) } & \text { (25) }\end{array}$

$\begin{array}{lllllllll}9 & 15 & 39 & 8 & 15 & 37 & 4 & 13 & 30\end{array}$

$\begin{array}{ccccccccc}9 & 15 & 39 & 8 & 15 & 37 & 4 & 13 & 30 \\ (24) & (20) & (19) & (15) & (13) & (14) & (12) & (13) & (13)\end{array}$

$g(y)=1-\exp \left\{-(y / 30)^{-4}\right\}:$

Method 1

Method 2

Method 3

Method 4

Method 5

$\begin{array}{ccccccccc}13 & 9 & 9 & 13 & 8 & 9 & 10 & 5 & 2 \\ (22) & (17) & (16) & (18) & (14) & (14) & (18) & (14) & (13) \\ 25 & 26 & 35 & 45 & 43 & 54 & 40 & 45 & 55\end{array}$

$\begin{array}{llllllll}(19) & (17) \quad(18) & (17) & (16) & \text { (21) } & \text { (23) } & \text { (24) } & \text { (23) }\end{array}$

$\begin{array}{lllllllll}15 & 11 & 7 & 14 & 12 & 14 & 11 & 11 & 10\end{array}$

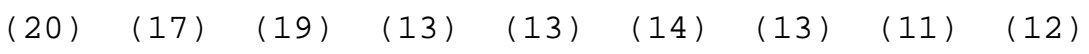

$\begin{array}{lllllllll}24 & 18 & 15 & 43 & 35 & 26 & 40 & 34 & 22\end{array}$

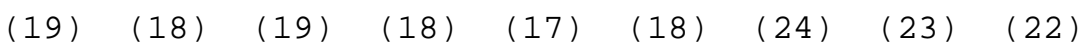

$\begin{array}{ccccccccc}14 & 17 & 37 & 16 & 20 & 45 & 12 & 17 & 36 \\ (19) & (17) & (19) & (14) & (13) & (14) & (14) & (12) & (12)\end{array}$

632

633 
634 Table 2. Percent bias of mean group size estimates, simulation set A. Coefficients of 635 variation of estimates, expressed as percentages, are shown in parentheses. Method a: 636 sample mean of recorded group sizes within $w$ of the line. Method b: sample mean of 637 true group sizes, detected groups within $w$ of the line only. Method c: estimated mean 638 based on a regression of the log of recorded group sizes on estimated probability of 639 detection. Method d: estimated mean based on a regression of the log of true sizes of 640 detected groups on estimated probability of detection.

641

642

643

644

645

646

647

648

649

650

651

652

653

654

655

656

657

658

659

660

661

662

663

664

665

666

667

668

669

670

671

\begin{tabular}{llllllllll}
\hline Mean group size & & 3 & & & 10 & & & 30 & \\
Half-group spread & $10 \mathrm{~m}$ & $25 \mathrm{~m}$ & $50 \mathrm{~m}$ & $10 \mathrm{~m}$ & $25 \mathrm{~m}$ & $50 \mathrm{~m}$ & $10 \mathrm{~m}$ & $25 \mathrm{~m}$ & $50 \mathrm{~m}$ \\
\hline
\end{tabular}
$g(y)=1-\exp \left\{-(y / 20)^{-2}\right\}$ :

Method a

$\begin{array}{lllllllll}-12 & -15 & -22 & -35 & -37 & -43 & -52 & -52 & -56\end{array}$

Method b

(8) (7)

(6)

(6)

(6)

(6)

(6)

(7)

(6)

Method

$\begin{array}{llll}10 & 11 & 9 & 5 \\ (6) & (6) & (5) & (4)\end{array}$

Method c

(6) (6)

(5)

(4)

4
$(4)$

$\stackrel{3}{(4)}$

(3)

21

$\begin{array}{llll}9 & 2 & -15 & 5\end{array}$

$\begin{array}{cccc}9 & 2 & -15 & 5 \\ (10) & (10) & (9) & (9)\end{array}$

$\begin{array}{ccccc}0 & -16 & -16 & -11 & -6 \\ (9) & (8) & (23) & (17) & (16)\end{array}$

Method d

$\begin{array}{ccccccccc}4 & 3 & 6 & 0 & 0 & 0 & -1 & 1 & 1 \\ (10) & (11) & (9) & (6) & (6) & (6) & (4) & (4) & (4)\end{array}$

$g(y)=1-\exp \left\{-(y / 30)^{-4}\right\}:$

Method a

$\begin{array}{ll}-4 & -7\end{array}$

$-7 \quad-16$

$-19$

$-23$

$-33$

$-34$

$-36$

$-45$

Method b

(8)

$\begin{array}{lll}6 & 7 & 9\end{array}$

4
$(4)$

3

3

(8)

(6) (6)

Method c

(7) (7) (5)

(10)

$\begin{array}{ccccccccc}9 & 5 & -9 & 23 & 14 & -8 & 23 & 14 & -5 \\ 10) & (10) & (9) & (10) & (10) & (11) & (16) & (16) & (15)\end{array}$

Method d

$\begin{array}{cc}\odot & 1 \\ (7) & (10)\end{array}$

672

673

674 
675 Table 3. Percent bias of density estimates for the five methods of estimation for 676 simulation set B. Coefficients of variation of estimates, expressed as percentages, are 677 shown in parentheses. See caption to Table 1 for methods.

678

679

\begin{tabular}{lccccccccc}
\hline Mean group size & & 3 & & & 10 & & & 30 \\
Half-group spread & $10 \mathrm{~m}$ & $25 \mathrm{~m}$ & $50 \mathrm{~m}$ & $10 \mathrm{~m}$ & $25 \mathrm{~m}$ & $50 \mathrm{~m}$ & $10 \mathrm{~m}$ & $25 \mathrm{~m}$ & $50 \mathrm{~m}$ \\
True density & 15 & 15 & 15 & 50 & 50 & 50 & 150 & 150 & 150 \\
\hline
\end{tabular}

$682 \quad g(y)=1-\exp \left\{-(y / 20)^{-2}\right\}$ :

683

684

685

686

687

688

689

690

691

692

693

694

695

Method 1

Method 2

Method 3

Method 4

Method 5

$\begin{array}{ccccccccc}11 & 5 & 3 & 11 & 1 & 5 & 4 & 11 & 8 \\ (31) & (25) & (23) & (39) & (29) & (19) & (29) & (27) & (20)\end{array}$

$\begin{array}{ccccccccc}-3 & 5 & 13 & -22 & -6 & 29 & -35 & -18 & 40 \\ (30) & (29) & (29) & (27) & (35) & (21) & (37) & (43) & (30)\end{array}$

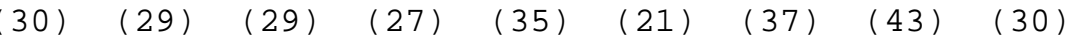

$\begin{array}{ccccccccc}6 & 2 & -2 & 1 & -2 & 3 & 1 & 1 & 3 \\ (25) & (21) & (24) & (15) & (19) & (14) & (13) & (15) & (13)\end{array}$

$\begin{array}{lllllllll}6 & -2 & -1 & -22 & -12 & 2 & -36 & -24 & 4\end{array}$

$\begin{array}{llllllll}(25) & (26) & (30) & (28) & \text { (35) } & \text { (24) } & \text { (38) } & \text { (40) }\end{array}$

$\begin{array}{lllllllll}10 & 29 & 67 & 3 & 20 & 77 & 3 & 15 & 59\end{array}$

$\begin{array}{lllllll}(24) \quad(24) \quad(30) & (16) & (23) & \text { (18) (14) (17) }\end{array}$

696

697

698

699

700

701

702

703

704

705

706

707

708

$g(y)=1-\exp \left\{-(y / 30)^{-4}\right\}$

Method 1

Method 2

$\left.\begin{array}{llll}(26) & (24) & (19) & (39\end{array}\right)$

7

5
$(19)$

9

$\begin{array}{cc}4 & 1 \\ (25) & (18)\end{array}$

$\begin{array}{lllllllll}17 & 25 & 37 & 17 & 25 & 61 & 1 & 22 & 62\end{array}$

Method 3

(24) (22) (23)

(25)

(24)

(24)

(26) (27) (22)

Method 4

$\begin{array}{ccccccccc}8 & 9 & 3 & 6 & 5 & 7 & 4 & 5 & 2 \\ (23) & (20) & (22) & (19) & (17) & (16) & (16) & (14) & (13)\end{array}$

$\begin{array}{lllllllll}16 & 15 & 11 & 17 & 16 & 19 & 0 & 11 & 21\end{array}$

$\begin{array}{llllllll}(24) \quad(23) \quad(20) & (28) & \text { (23) } & \text { (19) } & \text { (28) } & \text { (23) } & \text { (21) }\end{array}$

Method 5

$\begin{array}{lllllllll}11 & 29 & 67 & 11 & 26 & 79 & 7 & 22 & 63\end{array}$

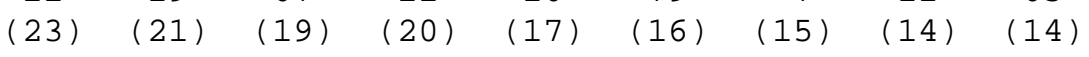


Fig. 1. Examples of survey design within a region for which an estimate of abundance is required. (a) Systematic random sample of lines that span the survey region. (b) Stratified systematic sample of lines that span the stratum. (c) Systematic sample of line segments. The design comprises the solid line segments. (d) Systematic sample of circuit transects.

(a)

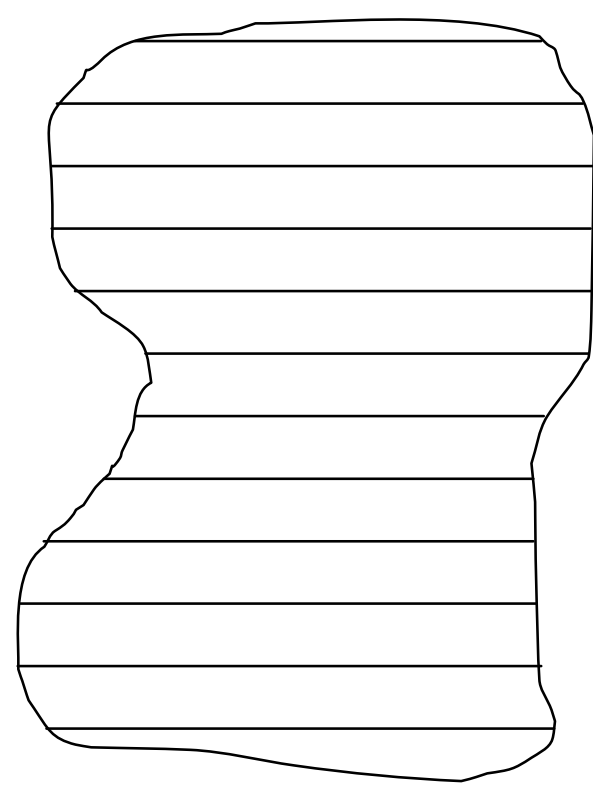

(c)

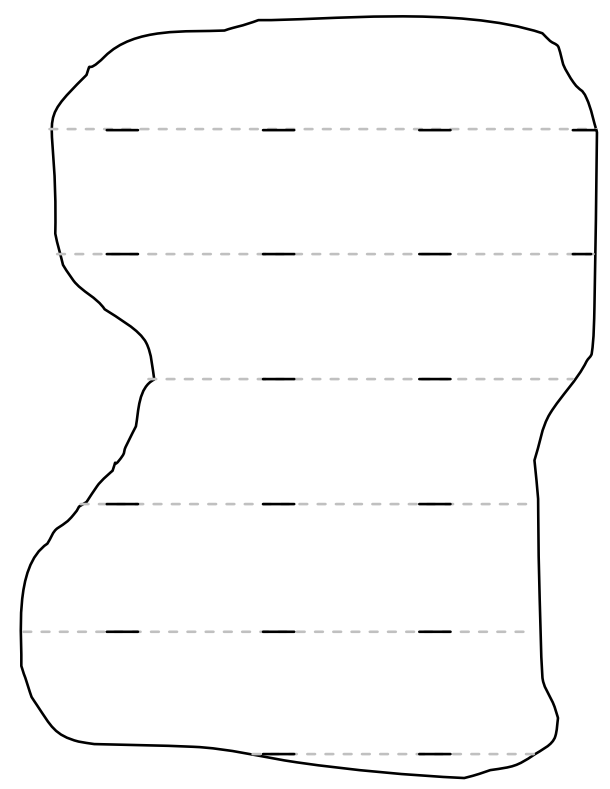

(b)

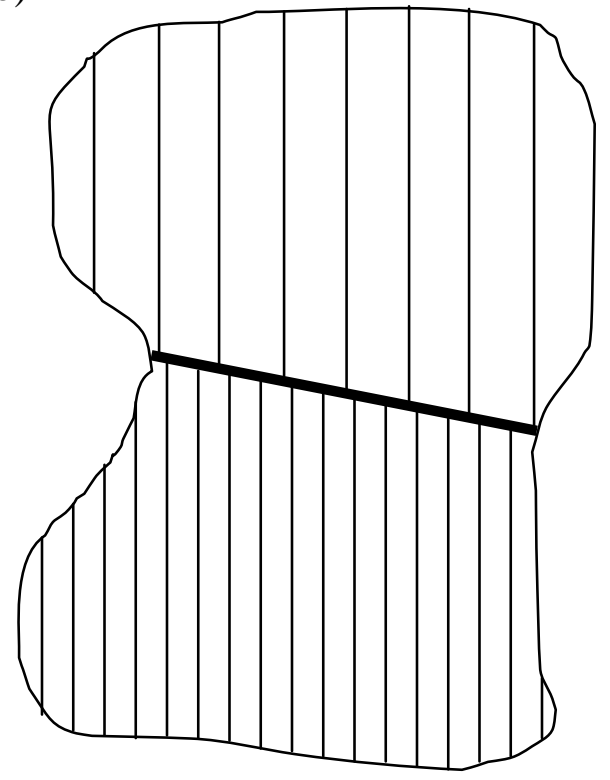

(d)

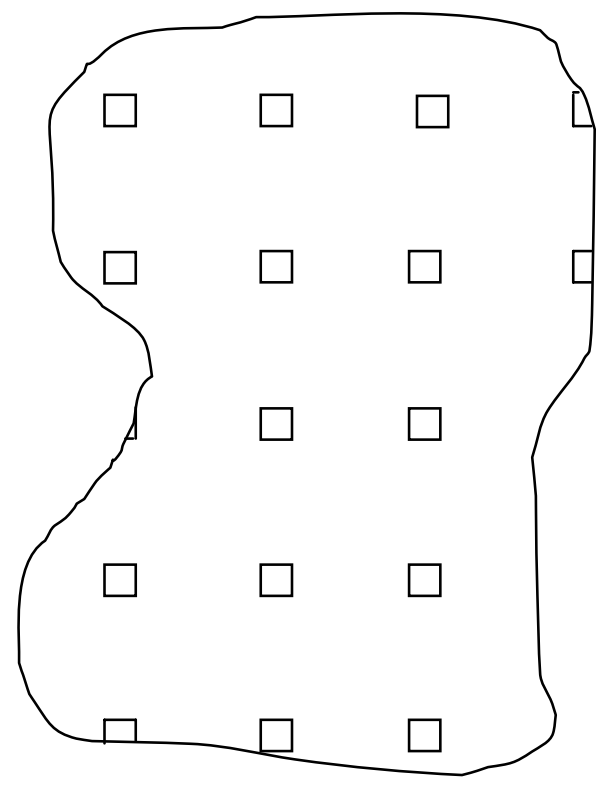

